\title{
Is attention really biased toward the last target location in visual search? Attention, response rules, distractors, and eye movements
}

\author{
Matthew D. Hilchey ${ }^{1}$ - Victoria Antinucci ${ }^{1} \cdot$ Dominique Lamy $^{2} \cdot$ Jay Pratt $^{1}$ \\ Published online: 22 February 2019 \\ (C) The Psychonomic Society, Inc. 2019
}

\begin{abstract}
The visual search and target-target cueing literatures have reached opposite conclusions about whether a shift of attention is biased toward or away from, respectively, previously attended target locations. In this article, we aimed to figure out why. The main differences between the two experimental approaches concern (1) the stimulus-response translation rules ("what" identification keypresses vs. "where" localization responses), (2) the amount of attention required in order to identify the target, and (3) distractor presence or absence. Experiment 1 tested the role of stimulus-response translation rules by requiring both an eye movement "where" response and a keypress "what" response to each target, in a typical search paradigm. Eye movements showed a bias away from the vicinity of the previous target, whereas keypresses showed a bias toward the previous target location, but only when the keypress response repeated. Experiment 2 removed the keypress identification requirement, to test whether reducing the amount of attention to the target would alter the eye movement bias; it did not. Experiment 3 removed the distractors, to test whether eliminating the potential for distractor location effects would alter the eye movement bias; it did, by accentuating the eye movement bias against the last target location. Collectively, the findings revealed that different stimulusresponse translation rules and distractor-processing requirements are the main reasons for the discrepancy, while demonstrating that shifts of attention intrinsically tend away from prior target locations. The findings are generally consistent with episodicretrieval and inhibited spatial-reorienting theories.
\end{abstract}

Keywords Visual search $\cdot$ Priming $\cdot$ Attention $\cdot$ Episodic memory $\cdot$ Inhibition of reutrn

Is it easier to find a target stimulus when its location repeats within a display? The answer to this simple question has proven surprisingly complicated. Findings from the visual search literature suggest that a target is identified more efficiently when its location repeats (e.g., Hilchey, Leber, \& Pratt, 2018a; Maljkovic \& Nakayama, 1996). However, findings from the target-target cueing literature, in which a localization response is made first to a cue and then later to a target, suggest that a target is localized less efficiently when its location repeats (e.g., Huffman, Hilchey, \& Pratt, 2018; Kwak \& Egeth, 1992). Both literatures claim that their findings reflect bias in the deployment of attention, either toward the previous

Matthew D. Hilchey

matthew.hilchey@utoronto.ca

1 Department of Psychology, University of Toronto, Toronto, Ontario, Canada

2 School of Psychological Sciences, Tel Aviv University, Tel Aviv, Israel target location (visual search) or away from it (cueing). What is responsible for this discrepancy?

The prior literature has identified three likely culprits, the first being a difference in stimulus-response translation rules (Tanaka \& Shimojo, 2000). In the search literature, each target is typically associated with a unique keypress response (discrimination tasks), whereas in the cueing literature, each target location is associated with a unique response (localization tasks). This difference is particularly important in episodic-retrieval theories (Hommel, 2004, 2007). According to such theories, whenever a response or location repeats, the location or response last associated with it, respectively, is retrieved. If there is a mismatch between the retrieved information and the required information, interference occurs, slowing down responding (Hilchey, Rajsic, Huffman, \& Pratt, 2017; Hommel, 1998; Hommel, Proctor, \& $\mathrm{Vu}, 2004)$. Thus, interference at this level can affect the discrimination but not the localization responses, because with the latter responses, neither repeating nor changing the target location can result in a partial location-response match.

Second, often confounded with the first difference, visual search studies typically require a fine-grained perceptual 
analysis of each target, whereas cueing studies do not. Indeed, search studies require choice responses to a target's identity (e.g., shape or color), whereas cueing studies require choice responses to the target location, irrespective of target identity. Accordingly, whether responses to previous target locations are facilitated may depend on the perceptual processing demands at the target location. The claim that the deployment of attention is biased to the previous target location only when a fine-grained perceptual analysis must be performed - and thus when focal attention is required - is consistent with this suggestion (e.g., Krummenacher, Müller, Zehetleitner, \& Geyer, 2009; Maljkovic \& Nakayama, 1996; Tanaka \& Shimojo, 1996, 2000; Yashar \& Lamy, 2010).

Third, search studies, by definition, embed their target in an array of distractors, and often the target location either repeats or changes to a distractor location. Typical cueing studies do not include distractors. This difference may be important, because presenting a target at a previous distractor location, relative to a previously vacant location, can slow down responding (e.g., Kumada \& Humphreys, 2002; Maljkovic \& Nakayama, 1996; Tipper, Brehaut, \& Driver, 1990). There are multiple interpretations of such findings, but in the rubric of episodic-retrieval theories (Neill \& Mathis, 1998; Neill, Valdes, \& Terry, 1995), they have been attributed to a conflict between the prior distractor location's response code (i.e., "do not respond") and the response code that is needed when a target later appears at it.

Here, we tested the relative contributions of the three possible factors for differential target location transition effects, by starting out with a visual search task that required a subtle discrimination of the target shape, and then modifying it until we were left with a target-target cueing approach that required neither a fine-grained perceptual analysis of the target shape nor distractor processing. Thus, the factors responsible for faster versus slower responses when the target location repeats relative to when it does not were determined through the progression of the experiments.

\section{Experiment 1}

We adapted the approach from the search literature that uses chipped diamond stimuli to stack the deck in favor of obtaining faster responses for target location repeats than for switches (e.g., Maljkovic \& Nakayama, 1996). The target color was either red or green, with three distractors taking on the nontarget color. Participants indicated the side on which the diamond was chipped by pressing one of two keys, and the target location randomly repeated or switched to a distractor location across displays.

Most importantly, participants were required to make an eye movement from a central fixation point to each target before the keypress. This method thus allows us to assess the eye movement initiation times and keypress response times to test whether the target was localized and identified, respectively, more efficiently when its location repeated in a visual search task that required the enactment of a fine-grained perceptual analysis of the target.

\section{Method}

Participants Seventeen undergraduates from the University of Toronto consented to participate. They received course credit or $\$ 10$ cash $(\mathrm{CDN})$. All participants had normal or correctedto-normal vision.

Stimulus and apparatus Eye movements were monitored by an EyeLink 1000 desktop mount eye tracker (ST Research, Ottawa, Ontario, Canada) with a temporal threshold of $1000 \mathrm{~Hz}$ and a spatial resolution of $0.01^{\circ}$ of visual angle. The velocity and acceleration thresholds for eye movements were set to $35.0 \%$ and $9500.0 \% \mathrm{~s}^{2}$. Stimuli were displayed on an 18-in. Dell P992 CRT monitor (Dell Computer, Round Rock, TX) with a refresh rate of $85 \mathrm{~Hz}$ and a $1,024 \times 768$ pixel resolution. Head position was stabilized by a chin- and headrest $57 \mathrm{~cm}$ from the monitor. Standard nine-point calibration and validation procedures were used until the average measurement error on gaze position was within a half degree. Keypresses were made with the spacebar (right thumb), "B" key (right index finger), or " $\mathrm{N}$ " key (right middle finger) on a QWERTY keyboard.

The fixation display consisted of a white fixation cross on a black background, located at screen center and subtending $0.5^{\circ} \times 0.5^{\circ}$. The cue-back display consisted of a white circle (radius $=0.25^{\circ}$ ) at the center of the screen. Each search display consisted of one uniquely colored red or green diamond $\left(2.0^{\circ}\right.$ $\times 2.0^{\circ}$ ) and three same-colored diamonds in the nontarget color. Each diamond was positioned $9.90^{\circ}$ away from the fixation cross to form an imaginary square $\left(7.0^{\circ} \times 7.0^{\circ}\right)$, and one corner of each diamond was cut $0.5^{\circ}$ inward.

Procedure The sequence of events is presented in Fig. 1. To begin each trial, the participant initiated a drift correction procedure by staring at the fixation cross and pressing the spacebar. Successful and unsuccessful drift corrections were signaled by tones. If drift correction was successful, a half second later the first search display appeared.

On the first search display, the participant made an eye movement to the uniquely colored diamond (the "target"). The participant then indicated whether the diamond was chipped on its left or right side ("B" keypress) or its top or bottom ("N" keypress). Once the eyes landed within $3^{\circ}$ of the target and a keypress was made, the display disappeared and the fixation cross transformed into a white circle, which we refer to as a cue-back, because it cued the eyes back to fixation. Once the return eye movement landed within $3^{\circ}$ of 


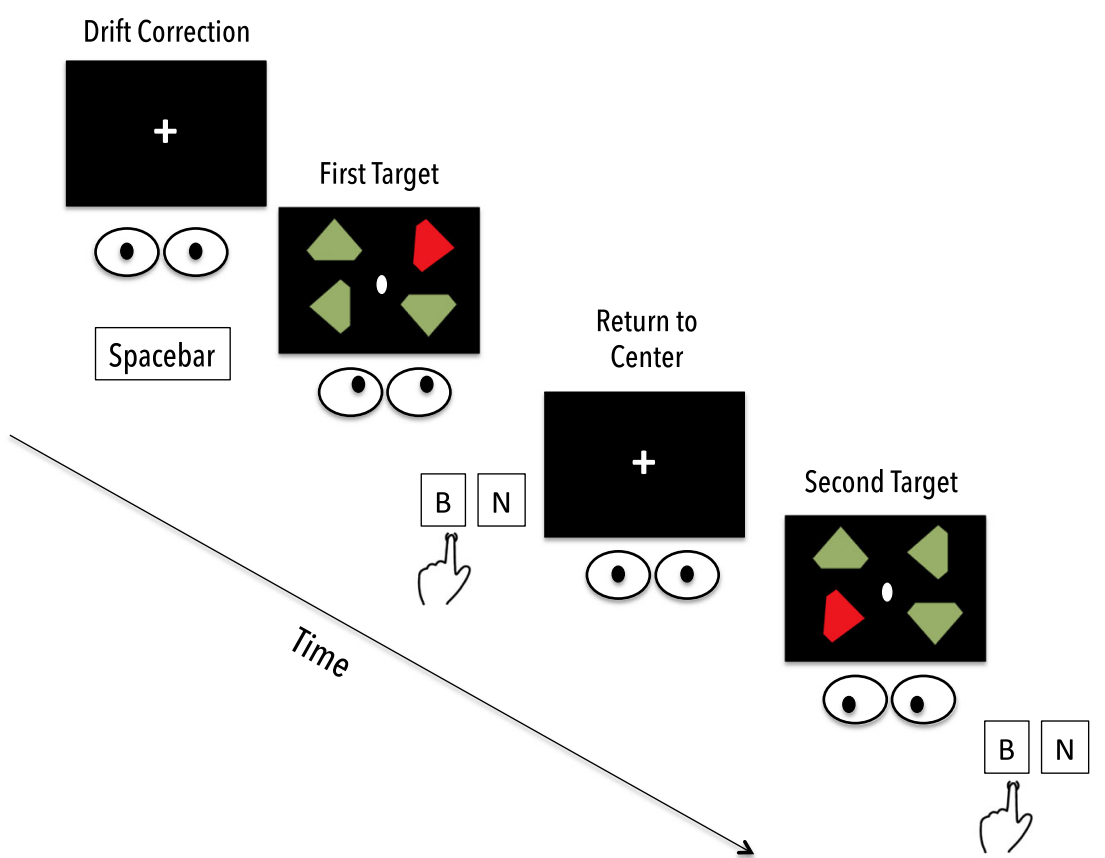

Fig. 1 A possible trial sequence in Experiment 1. Each target is looked at and then identified with one of two keypresses, after which the eye is reoriented to screen center. Here, the distance between the two target

center, the cue-back transformed into a fixation cross. The participant stared at fixation for $1 \mathrm{~s}$, then the second search display appeared. The task on the second search display was the same as that on the first. Once the eyes landed within $3^{\circ}$ of the target and the keypress was made, all stimuli disappeared, signaling the end of the trial.

If an incorrect keypress was made, an error message appeared at the end of the trial, with a reminder of the correct keypress-response mappings. If eight or more eye movements were made, the trial immediately ended with an error message. Participants had to acknowledge these messages with the spacebar. There was a 1-s blank intertrial interval.

Participants were told that there was no relationship between successive targets. They were also told that an eye movement was required to each target before the keypress. They were told that each trial could be completed with only three eye movements. Each participant performed 20 practice trials and then 384 experimental trials.

\section{Results}

Exclusions One participant was replaced because he made more than eight eye movements on over $25 \%$ of trials. Of the remaining data, $7.94 \%$ of the trials were excluded for having more than eight eye movements, $0.4 \%$ because the keypress to the target in the second search display occurred before the eye movement, $0.6 \%$ because the eyes were not within $5^{\circ}$ of fixation at the time of the second display, $17.71 \%$ because the first eye movement to the second display locations is maximal (i.e., change diagonal), the target color has repeated, and so has the manual response

did not land within $5^{\circ}$ of the target (an error that was frequent when the target color switched; see Table 1), $3.61 \%$ because a keypress error was made, and $0.29 \%$ and $0.26 \%$, respectively, for exceptionally slow eye movements ( $>1 \mathrm{~s}$ ) and keypresses $(>2.5 \mathrm{~s})$ to the second display. Next, $z$-scores were computed for each participant for both saccadic and keypress response times to detect outliers (trials containing $z$-scores $>$ or $<3$ were excluded as outliers) on the second display; $1.1 \%$ and $1.2 \%$ of trials were excluded as outliers, respectively.

Saccadic responses The mean saccadic response times (SRTs) and error rates are presented in Table 1. The SRTs were analyzed with a 4 (target location transition: same, change

Table 1 Mean saccadic response times (ms; line 1) and saccade landing error rates $(\%$; line 2$)$ in Experiment 1 for all combinations of target color and location transition

\begin{tabular}{lll}
\hline & Repeat Color & Switch Color \\
\hline Same location & $293(100)$ & $356(132)$ \\
Change vertical & $8.48(4.47)$ & $30.47(13.73)$ \\
& $296(97)$ & $355(115)$ \\
Change horizontal & $10.80(4.41)$ & $34.10(19.16)$ \\
Change diagonal & $294(103)$ & $344(107)$ \\
& $7.90(4.15)$ & $32.86(19.49)$ \\
& $283(91)$ & $341(116)$ \\
& $6.65(6.22)$ & $26.58(12.42)$ \\
\hline
\end{tabular}

Standard deviations are in parentheses. 
vertical, change horizontal, or change diagonal) $\times 2$ (target color repetition: repeat or switch) repeated measures analysis of variance (ANOVA). We found an effect of target location transition, $F(3,45)=4.453, p<.01, \eta_{\mathrm{p}}{ }^{2}=.2289$. SRTs were fastest when the distance between the two targets was greatest (i.e., on a diagonal; see Fig. 2, left panel). There was also an effect of target color repetition, $F(1,15)=75.77, p<.01, \eta_{\mathrm{p}}{ }^{2}=$ .8347. SRTs were faster when the target color repeated ( 292 $\mathrm{ms})$ than when it switched $(349 \mathrm{~ms})$. No interaction emerged, $F<1$. There were no concerns about speed-accuracy tradeoffs, because the error rates tended in the same direction as the SRT data (see Table 1).

Manual responses The mean manual response times (MRTs) and error rates are presented in Table 2 and were analyzed with a 4 (target location transition) $\times 2$ (target color repetition) $\times 2$ (target response repetition: repeat or switch) repeated measures ANOVA. The effect of target location transition was marginal, $F(3,45)=2.153, p=.107, \eta_{\mathrm{p}}^{2}=.1255$, with MRTs being fastest when the target location repeated (849 $\mathrm{ms} ; 871,863$, and $861 \mathrm{~ms}$ for change vertical, horizontal, and diagonal, respectively). We observed an effect of target color repetition, $F(3,45)=86.26, p<.01, \eta_{\mathrm{p}}^{2}=.8519$, with faster keypresses when the target color repeated $(806 \mathrm{~ms})$ than when it switched (916 ms). The effect of target response repetition was not significant $(F<1)$.

Target location transition and target response repetition interacted, $F(3,45)=4.874, p<.01, \eta_{\mathrm{p}}{ }^{2}=.2452$ (see Fig. 2, right panel). Repeating the target location and response led to

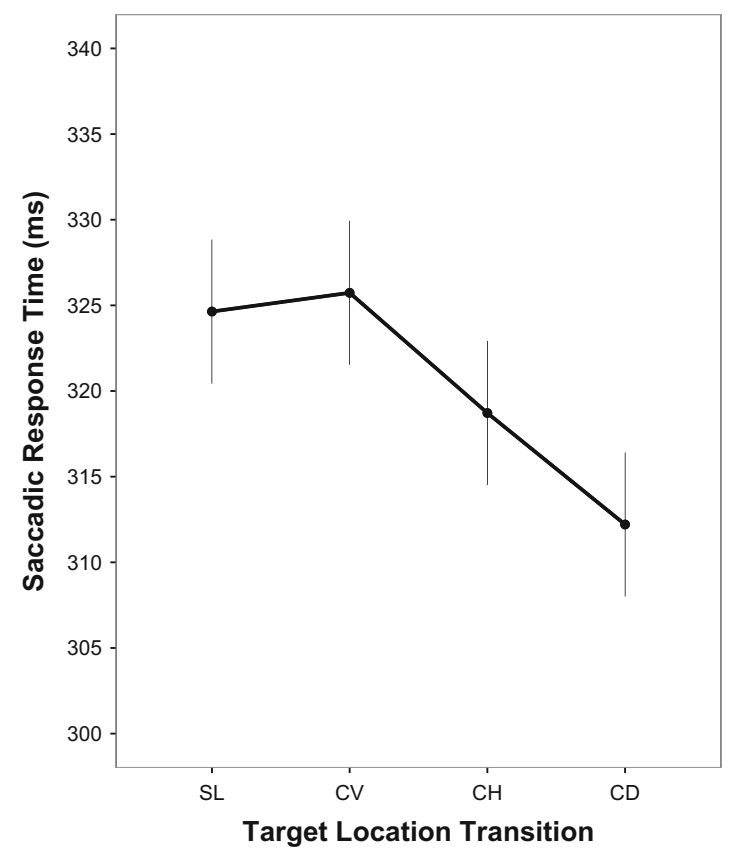

Fig. 2 (Left) Effects of target location transitions on eye movement response times in Experiment 1. (Right) The interaction between target location transition and response repetition for keypress discrimination response times. Errors bars show protected half Fisher least significant especially fast reactions relative to all other transitions. The only other interaction to approach significance was between target color repetition and target response repetition, $F(1,15)$ $=4.245, p=.0571, \eta_{\mathrm{p}}{ }^{2}=.2206$ : The effect of repeating the target color was larger when the response repeated $(123 \mathrm{~ms})$ than when it switched $(95 \mathrm{~ms})$. No other interactions were significant $\left(p \mathrm{~s}>.432, \eta_{\mathrm{p}}{ }^{2} \mathrm{~s}<.0587\right)$.

Keypress errors to the second display (\% error) were evaluated for speed-accuracy trade-offs with an ANOVA involving the same factors used above. The effects of target location transition, $F<1$, and target color repetition, $F(1,15)=2.344$, $p=.147, \eta_{\mathrm{p}}^{2}=.1351$, were not significant. The effect of target response repetition was significant, $F(1,15)=6.267, p=.024$, $\eta_{\mathrm{p}}{ }^{2}=.2947$, with more accurate responses for response repeats (2.50\%) than for switches $(4.00 \%)$.

We found two two-way interactions. One was between target color repetition and target response repetition, $F(1,15)=$ 23.20, $p<.01, \eta_{\mathrm{p}}{ }^{2}=.4877$ : Generally speaking, more errors were made when the color and response both repeated $(3.42 \%)$ or switched $(6.10 \%)$ than when only the color $(1.91 \%)$ or the response $(1.57 \%)$ switched. More critically, the other interaction was between target location transition and target response repetition, $F(3,45)=14.28, p<.01, \eta_{\mathrm{p}}^{2}$ $=.6074$ : For response repeats, repeating the target location led to more errors $(5.01 \%)$ than in all other location transitions, $F(3,45)=6.23, p<.01, \eta_{\mathrm{p}}{ }^{2}=.2935$, with the other location transitions yielding similar error rates $(F<1$; change vertical $=$ $2.04 \%$; change horizontal $=1.15 \%$; change diagonal $=$ $2.04 \%$ ). Thus, the especially fast responses for location and

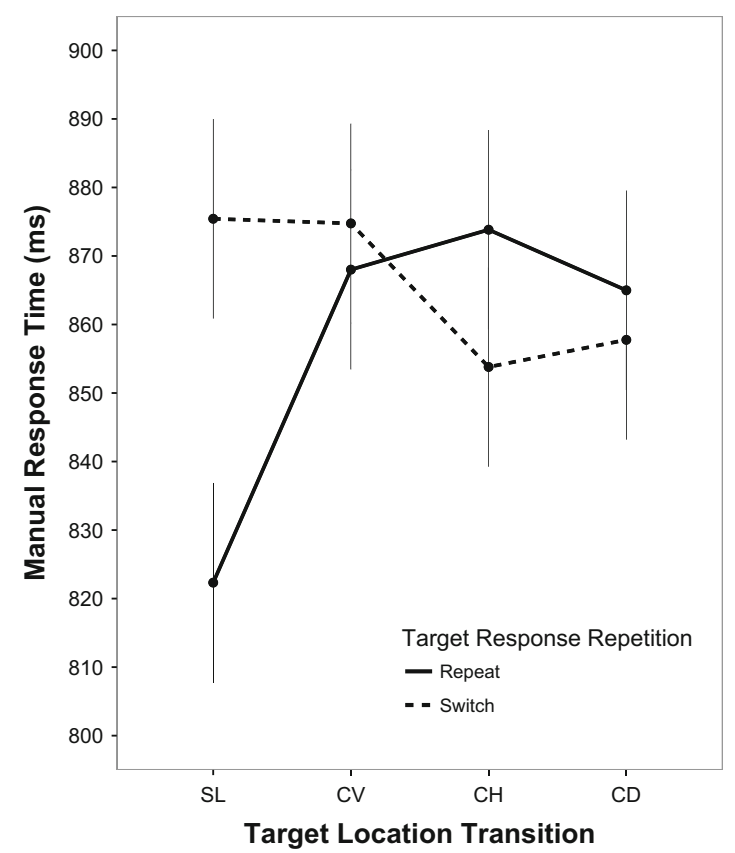

differences computed from the mean squared error term of the effect (left) and interaction (right); overlap signifies a nonsignificant simple effect. SL = same location $; \mathrm{CV}=$ change vertical $; \mathrm{CH}=$ change horizontal $; \mathrm{CD}=$ change diagonal 
Table 2 Mean manual response times (ms; line 1) and manual error rates (\%; line 2) in Experiment 1 for all combinations of target color, target location transition, and response repetition

\begin{tabular}{|c|c|c|c|c|}
\hline & \multicolumn{2}{|c|}{ Repeat Response } & \multicolumn{2}{|c|}{ Switch Response } \\
\hline & Repeat Color & Switch Color & Repeat Color & Switch Color \\
\hline \multirow[t]{2}{*}{ Same location } & $770(234)$ & $884(236)$ & 817 (207) & 947 (272) \\
\hline & $5.76(6.53)$ & $4.26(6.56)$ & $1.14(2.46)$ & $3.35(4.88)$ \\
\hline \multirow[t]{2}{*}{ Change vertical } & $800(229)$ & $955(237)$ & $835(233)$ & $939(257)$ \\
\hline & $2.49(4.54)$ & $1.59(3.71)$ & $1.00(2.23)$ & $6.64(8.84)$ \\
\hline \multirow[t]{2}{*}{ Change horizontal } & $802(227)$ & $943(255)$ & $823(233)$ & $900(241)$ \\
\hline & $2.30(4.67)$ & $0.00(0.00)$ & $3.52(4.30)$ & $8.30(6.93)$ \\
\hline \multirow[t]{2}{*}{ Change diagonal } & $798(233)$ & $933(245)$ & $784(208)$ & $914(272)$ \\
\hline & $3.15(4.52)$ & $0.42(1.67)$ & $2.01(3.24)$ & $6.11(6.14)$ \\
\hline
\end{tabular}

Standard deviations are in parentheses.

response repeats at least partly reflected a speed-accuracy trade-off. For response switches, repeating the target location led to fewer errors $(2.22 \%)$ than all other location transitions, $F(3,45)=4.604, p<.01, \eta_{\mathrm{p}}{ }^{2}=.2348$, with the other transitions yielding roughly similar error rates, $F(2,30)=2.76, p=$ $.080, \eta_{\mathrm{p}}{ }^{2}=.1552$ (change vertical $=3.81 \%$; change horizontal $=5.91 \%$; change diagonal $=4.06 \%$ ). The remaining interactions were not significant, $F \mathrm{~s}<1$.

\section{Discussion}

Turning first to the MRTs, we achieved our goal of creating a situation in which responses were faster for targets at repeated locations. Importantly, this was qualified by an interaction with response repetition, such that faster responses only occurred when both the target location and the response repeated. This is consistent with recent findings showing that postattentional orienting processes factor into the keypress identification responses (e.g., Gokce, Geyer, Finke, Müller, \& Töllner, 2014; Hilchey, Leber, \& Pratt, 2018a; Hilchey, Rajsic, Huffman, Klein, \& Pratt, 2018b), as is suggested by episodic-retrieval theories postulating interference whenever (1) a target appears at a prior distractor location (Neill \& Mathis, 1998), (2) a target location repeats but the response changes, or (3) the response repeats but the target location changes (Hommel, 2004). Accordingly, the only location transition not suffering from some amount of interference would be target location and response repeat, as we found here. However, interestingly and unexpectedly, the error rate analysis suggested a speedaccuracy trade-off. To account for this trade-off, retrieval theories must assume that at least some forms of interference can delay decision-making processes without affecting the quality of the visual signals or stimulus-response translation.

With the MRTs being an unreliable indicator of whether attention is biased away or toward repeated locations, we turn to the SRTs. Here the data were quite clear: SRTs were never any faster when the target location repeated instead of switched, and were indeed slower when the target location/ hemifield repeated relative to the change diagonal transition. Thus, not even in a visual search situation that requires finegrained perceptual analysis of the target is there evidence that targets are located more quickly when their location repeats. Instead, the SRT data reveal a weak bias against the last target location/hemifield, generally consistent with the idea that a shift of attention is biased against the vicinity of a prior target (Klein \& Redden, 2018; Vaughan, 1984).

As a secondary point, the MRTs and SRTs both reveal bias toward the last target color, consistent with other research (e.g., Moher \& Song, 2016).

\section{Experiment 2}

An unusual effect in the SRTs of Experiment 1 was that they were equally slow, irrespective of whether the target location or hemifield repeated. Why weren't responses especially slow when the target location repeated, as was shown by previous eye movement research in target-target cueing paradigms (e.g., Jayaraman, Klein, Hilchey, Patil, \& Mishra, 2016)? It could still be the case that performing a fine-grained perceptual analysis of the target required focal attention, which produced some amount of facilitation at the prior target location that added with any inhibition that was generated by orienting to it, as was suggested by Maljkovic and Nakayama (1996). To test this possibility, we replicated Experiment 1 but eliminated the need to discriminate the target's shape once it was looked at. If this kind of focal attention biased subsequent shifts of attention toward the last target location, thus offsetting some of the inhibition at that location, then by removing this focal-attention requirement, we should be able to show that SRTs become especially slow when the target location repeats. 


\section{Method}

Participants Sixteen different undergraduates at the University of Toronto consented to participate for course credit or cash. All had normal or corrected-to-normal vision.

Apparatus, stimuli, and procedure These were similar to those elements of Experiment 1, with two exceptions: (1) Keypress responses to targets were no longer required, and (2) participants were required to maintain their eyes at the target location for 500 or $700 \mathrm{~ms}$ before the search array disappeared, which ensured similar exposure durations for the search displays between experiments.

\section{Results}

We excluded $8.45 \%$ of trials for having more than eight eye movements, $0.20 \%$ because the eyes were not within $5^{\circ}$ of fixation at the time of the second search display, $11.90 \%$ because the first eye movement to the second search display did not land within $5^{\circ}$ of the target, and $1.1 \%$ for SRT outliers.

The SRTs appear in Table 3 and were analyzed with a 4 (target location transition) $\times 2$ (target color repetition) repeated measures ANOVA. There was an effect of target location transition, $F(3,45)=4.854, p<.01, \eta_{\mathrm{p}}{ }^{2}=.2445$ : SRTs were fastest when the separation between the two targets was greatest (see Fig. 3). We also observed an effect of target color repetition $F(1,15)=196.00, p<.01, \eta_{\mathrm{p}}{ }^{2}=.9289$, with faster SRTs when the target color repeated $(268 \mathrm{~ms})$ instead of changed $(302 \mathrm{~ms})$. The interaction was not significant, $F<$ 1. There were no concerns about speed-accuracy trade-offs.

\section{Comparison between Experiments 1 and 2}

To directly assess whether orienting was affected by the requirement to discriminate the target's shape, we conducted the same ANOVA on SRTs as in Experiment 1, except that we

Table 3 Mean saccadic response times (ms; line 1) and saccadic landing error rates (\%; line 2$)$ in Experiment 2 for all combinations of target color and location transition

\begin{tabular}{lll}
\hline & Repeat Color & Switch Color \\
\hline Same location & $268(57)$ & $303(59)$ \\
& $6.48(7.91)$ & $20.85(12.40)$ \\
Change vertical & $274(62)$ & $305(58)$ \\
Change horizontal & $7.32(8.57)$ & $23.24(12.76)$ \\
Change diagonal & $268(57)$ & $302(58)$ \\
& $6.63(5.72)$ & $21.47(12.25)$ \\
& $262(49)$ & $298(60)$ \\
& $4.00(3.62)$ & $17.34(12.46)$ \\
\hline
\end{tabular}

Standard deviations are in parentheses. included experiment as a between-subjects factor. No effect of experiment emerged, $F(1,30)=1.348, p=.255, \eta_{\mathrm{p}}{ }^{2}=.0430$; no interaction between target location transition and experiment, $F(3,90)=1.023, p=.386, \eta_{\mathrm{p}}{ }^{2}=.0330$; and no threeway interaction, $F<1$. However, there was an interaction between experiment and target color repetition, $F(1,30)=$ 11.04, $p<.01, \eta_{\mathrm{p}}^{2}=.2689$ : Repeating the target color sped up SRTs more in Experiment $1(57 \mathrm{~ms})$ than in Experiment 2 (34 ms), with the former experiment requiring discrimination of the target shape.

\section{Discussion}

Once again, SRTs were slowest when the target hemifield repeated. Thus, the requirement to perform a fine-grained perceptual analysis of the target shape after the shift in gaze did not determine target location transition effects. As a secondary point, this focal attention requirement did amplify the tendency to look for the target's color in the next search display.

\section{Experiment 3}

Because taking away the shape discrimination requirement did not restore the typical SRT pattern, we now turned to the role of distractors. Specifically, maybe partial overlap between a distractor location's response code and a target location's response code led to some amount of interference, as would occur when a target appeared at a prior distractor location. Any such interference would help offset any inhibition at the target location caused by shifting attention to it. This possibility was evaluated by removing the distractors. If in the earlier experiments there was a gradient of inhibition at the prior target location plus interference whenever a target appeared at a prior distractor location, removing the distractors should be sufficient to eliminate that interference, thereby revealing especially slow SRTs whenever the target location repeated.

\section{Method}

Participants Sixteen different undergraduates at the University of Toronto consented to participate for course credit or cash. All had normal or corrected-to-normal vision.

Apparatus, stimuli, and procedure These were similar to those elements of Experiment 2, except that the target displays no longer contained distractors.

\section{Results}

We excluded $5.94 \%$ of trials for more than eight eye movements, $1.66 \%$ because the eyes were not within $5^{\circ}$ of fixation at the time of the second target display, $2.77 \%$ because the first 


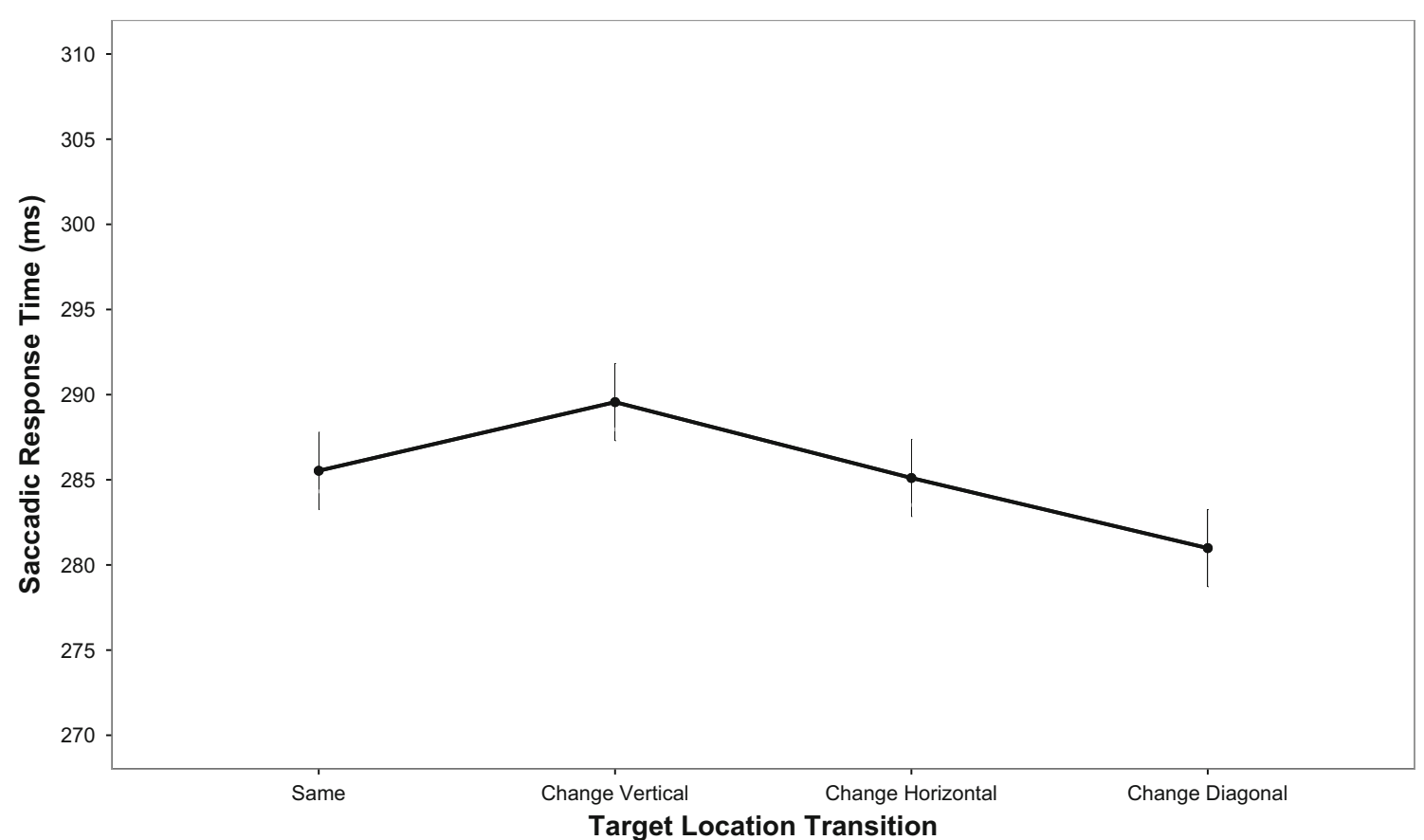

Fig. 3 Effects of target location transitions on eye movements in Experiment 2. Errors bars are protected half Fisher least significant differences computed from the mean squared error term of the effect; overlap signifies a nonsignificant simple effect

eye movement to the second target did not land within $5^{\circ}$, $0.02 \%$ for exceptionally slow $(>1 \mathrm{~s})$ or anticipatory $(<80$ $\mathrm{ms}$ ) eye movements to the second target display, and $1.2 \%$ for SRT outliers.

Mean SRTs appear in Table 4 and were analyzed with a 4 (target location transition) $\times 2$ (target color repetition) repeated measures ANOVA. We found an effect of target location transition, $F(3,45)=10.86, p<.01, \eta_{\mathrm{p}}{ }^{2}=$ .4200: SRTs were especially slow when the target location repeated relative to all other transitions, with the change vertical transition being the next slowest (see Fig. 4). This was not a speed-accuracy trade-off (see Table 4). There was no effect of target color repetition and no interaction, $F_{\mathrm{S}}<1$.

Table 4 Mean saccadic response times (ms; line 1) and saccadic landing errors (\%; line 2) in Experiment 3 for all combinations of target color and location repetition

\begin{tabular}{lll}
\hline & Repeat Color & Switch Color \\
\hline Same location & $205(57.59)$ & $206(60.86)$ \\
Change vertical & $3.40(2.76)$ & $3.13(3.41)$ \\
Change horizontal & $198(61.50)$ & $198(60.94)$ \\
& $3.67(4.53)$ & $2.05(2.32)$ \\
Change diagonal & $192(60.50)$ & $195(60.25)$ \\
& $3.76(3.35)$ & $2.73(3.91)$ \\
& $195(59.09)$ & $196(57.90)$ \\
\hline
\end{tabular}

Standard deviations are in parentheses.

\section{Comparison between Experiments 2 and 3}

To directly assess whether orienting was influenced by distractor presence, we conducted the same ANOVA on SRTs as in Experiment 2, except that we included experiment (Exp. 2 [distractors] vs. Exp. 3 [no distractors]) as a between-subjects factor. The effect of experiment was significant, $F(1,30)=26.36, p<.01, \eta_{\mathrm{p}}{ }^{2}=.4677$, with faster SRTs in Experiment 3 (198 $\mathrm{ms})$ than in Experiment 2 (285 ms). More importantly, we observed an interaction between target location transition and experiment, $F(3,90)=5.527, p<.01, \eta_{\mathrm{p}}{ }^{2}=.1556$. In Experiment 2, SRTs were especially slow when the target hemifield repeated relative to the change diagonal condition (Fig. 3). In Experiment 3, SRTs were especially slow whenever the target location repeated (Fig. 4).

There was also an interaction between experiment and target color repetition, $F(1,30)=138.70, p<.01, \eta_{\mathrm{p}}{ }^{2}=.8221$. Target color repetition had no apparent effect on SRTs in Experiment 3 (1 ms), whereas it speeded SRTs in Experiment 2 (34 ms). The three-way interaction was not significant, $F<1$.

\section{Discussion}

Distractor removal, and thus returning to the targettarget cueing paradigm, restored the typical target location transition effects: The slowest SRTs occurred specifically when the target location repeated. These findings suggest that a cost was associated with presenting a target at a prior distractor location, which is consistent 


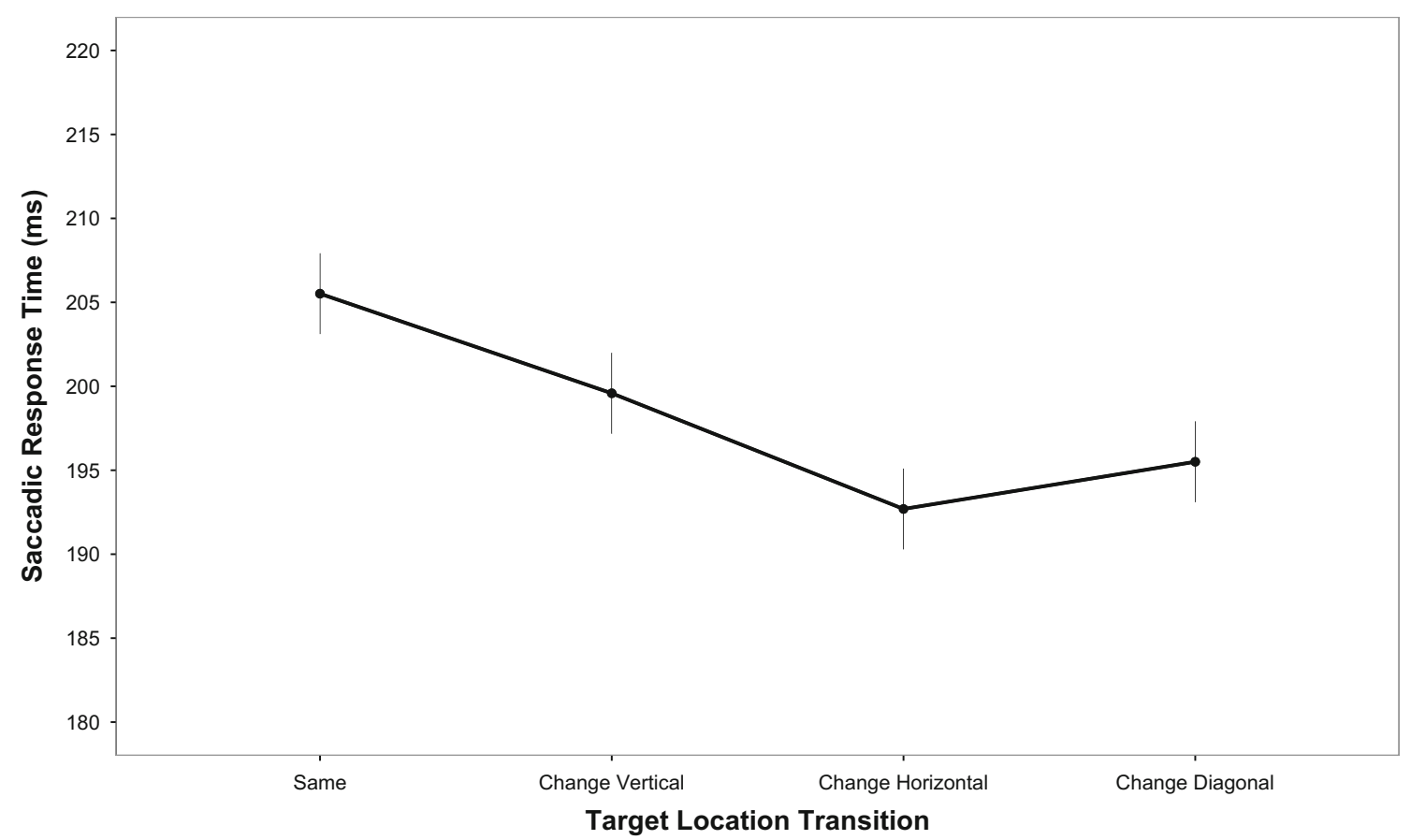

Fig. 4 Effect of repeating the target location on eye movements in Experiment 3. Errors bars are half Fisher least significant differences computed from the mean squared error term of the effect; overlap signifies a nonsignificant simple effect

with episodic-retrieval theories. ${ }^{1}$ Theoretically, if this cost could be made larger than the bias against the last target location, there would be a net bias in favor of the last target location. As a secondary point, the previously observed advantage for repeating the target color vanished, as was expected, given that paying attention to color was no longer necessary to find the target (e.g., Goolsby \& Suzuki, 2001; Huffman et al., 2018).

\section{General discussion}

How is it that two literatures (visual search and target-target cueing), using very similar paradigms, have arrived at opposite conclusions about whether attention is facilitated or inhibited to prior target locations? Having identified three differences between these literatures-the stimulus-response translation rules, the requirement to perform a fine-grained

\footnotetext{
${ }^{1}$ We thank an anonymous reviewer for suggesting an alternative interpretation. The difference between experiments could be at least partly explained by an asymmetry in the amount of perceptual analysis that was required before the target could be localized. In Experiment 3, it was not necessary to distinguish the target from the distractor colors in order to produce the correct eye movement. In Experiment 2, it was. Accordingly, the comparison between Experiments 2 and 3 could be taken to suggest that the requirement to perceptually analyze a target before it is localized behaviorally, but not after (cf. Exps. 1 and 2), is a determinant of the location transition effects. Taking this a step further, hypothetically, if the target were even more difficult to distinguish from the distractors, any eye movement bias toward the last target location could be enhanced to outweigh the bias against it.
}

perceptual analysis of the target, and distractor presencewe systematically tested them across three experiments.

The first experiment showed that target location transition effects were different depending on the stimulus-response translation rules. SRTs were slower when the target location/hemifield repeated as compared to switching to the mirror-opposite target location, a result consistent with shifts of attention being biased against the vicinity of an earlier target. Contrasting with these findings, MRTs were generally faster when the target location repeated, but only when the response also repeated, a result consistent with episodicretrieval theories, which posit interference whenever this is a partial mismatch between a current and prior stimulus location and response. Accordingly, the only location transition not suffering from some amount of proactive interference would be the target location with a response repeat.

Whether a fine-grained perceptual analysis of the target was needed in order to make a keypress (Exp. 1) or was not (Exp. 2) did not alter the eye movement bias against the vicinity of the last target. Whether there were distractors (Exp. 2) or were not (Exp. 3) did alter this eye movement bias. With distractors (Exp. 2), eye movements were slowest whenever the target hemifield repeated, regardless of whether the target location repeated. Without distractors, eye movements were slowest specifically when the target location repeated. Simply, there was a cost associated with a target appearing at a prior distractor location, consistent with episodic-retrieval theories postulating proactive interference between a past "do not respond" distractor location code and a required target location response code. 
Collectively, the SRT data are consistent with research showing slower eye movements when the target location repeats rather than switching to a more distant location, which is observed with distractors (e.g., Bichot \& Schall, 2002; Tanaka \& Shimojo, 1996), without distractors (Hilchey, Rajsic, et al., 2018b; Vaughan, 1984), and, as was also shown here, regardless of whether a finegrained perceptual analysis of the target is needed, once it is looked at. There is simply no direct evidence that eye movements, which we have evaluated for orienting bias, are biased overall in favor of the prior target location in simple visual search. Ultimately, the discrepancy between the literatures results from a combination of different stimulus-response rules and distractors. The SRT and MRT target location transition data in this article are consistent with a combination of episodic retrieval and inhibited spatial reorienting theories. Any link between performing a fine-grained perceptual analysis (i.e., focal attention) and biased reorienting to the last target location in visual search is either wrong or is not yet necessitated by the data.

Author note M.D.H. was supported by a Natural Sciences and Engineering Research Council of Canada (NSERC) postdoctoral fellowship. J.P. was supported by NSERC Discovery Grant 480593.

Publisher's note Springer Nature remains neutral with regard to jurisdictional claims in published maps and institutional affiliations.

\section{References}

Bichot, N. P., \& Schall, J. D. (2002). Priming in macaque frontal cortex during popout visual search: Feature-based facilitation and locationbased inhibition of return. Journal of Neuroscience, 22, 4675-4685. https://doi.org/10.1523/JNEUROSCI.22-11-04675.2002

Gokce, A., Geyer, T., Finke, K., Müller, H. J., \& Töllner, T. (2014). What pops out in positional priming of pop-out: Insights from eventrelated EEG lateralizations. Frontiers in Psychology, 5, 688. https://doi.org/10.3389/fpsyg.2014.00688

Goolsby, B. A., \& Suzuki, S. (2001). Understanding priming of colorsingleton search: Roles of attention at encoding and "retrieval". Perception \& Psychophysics, 63, 929-944. https://doi.org/10. 3758/BF03194513

Hilchey, M. D., Leber, A. B., \& Pratt, J. (2018a). Testing the role of response repetition in spatial priming in visual search. Attention, Perception, \& Psychophysics, 80, 1362-1374.

Hilchey, M. D., Rajsic, J., Huffman, G., Klein, R. M., \& Pratt, J. (2018b). Dissociating orienting biases from integration effects with eye movements. Psychological Science, 29, 328-339. https://doi.org/ 10.1177/0956797617734021

Hilchey, M. D., Rajsic, J., Huffman, G., \& Pratt, J. (2017). Intervening response events between identification targets do not always turn repetition benefits into repetition costs. Attention, Perception, \& Psychophysics, 79, 807-819. https://doi.org/10.3758/s13414-0161262-9
Hommel, B. (1998). Event files: Evidence for automatic integration of stimulus-response episodes. Visual Cognition, 5, 183-216. https:// doi.org/10.1080/713756773

Hommel, B. (2004). Event files: Feature binding in and across perception and action. Trends in Cognitive Sciences, 8, 494-500. https://doi. org/10.1016/j.tics.2004.08.007

Hommel, B. (2007). Feature integration across perception and action: Event files affect response choice. Psychological Research, 71, 42-63.

Hommel, B., Proctor, R. W., \& Vu, K.-P. L. (2004). A feature-integration account of sequential effects in the Simon task. Psychological Research, 68, 1-17. https://doi.org/10.1007/s00426-003-0132-y

Huffman, G., Hilchey, M. D., \& Pratt, J. (2018). Feature integration in basic detection and localization tasks: Insights from the attentional orienting literature. Attention, Perception, \& Psychophysics, 80, 1333-1341. https://doi.org/10.3758/s13414-018-1535-6.

Jayaraman, S., Klein, R. M., Hilchey, M. D., Patil, G. S., \& Mishra, R. K. (2016). Spatial gradients of oculomotor inhibition of return in deaf and normal adults. Experimental Brain Research, 234, 323-330.

Klein, R. M., \& Redden, R. S. (2018). Two "inhibitions of return" bias orienting differently. In T. L. Hubbard (Ed)., Spatial biases in perception and cognition (pp. 295-306). New York, NY: Cambridge University Press.

Krummenacher, J., Müller, H. J., Zehetleitner, M., \& Geyer, T. (2009). Dimension-and space-based intertrial effects in visual pop-out search: Modulation by task demands for focal-attentional processing. Psychological Research, 73, 186-197.

Kumada, T., \& Humphreys, G. W. (2002). Cross-dimensional interference and cross-trial inhibition. Perception \& Psychophysics, 64 , 493-503. https://doi.org/10.3758/BF03194720

Kwak, H. W., \& Egeth, H. (1992). Consequences of allocating attention to locations and to other attributes. Perception \& Psychophysics, 51, 455-464.

Maljkovic, V., \& Nakayama, K. (1996). Priming of pop-out: II. The role of position. Perception \& Psychophysics, 58, 977-991. https://doi. org/10.3758/BF03206826

Moher, J., \& Song, J. H. (2016). Target selection biases from recent experience transfer across effectors. Attention, Perception, \& Psychophysics, 78, 415-426.

Neill, W. T., \& Mathis, K. M. (1998). Transfer-inappropriate processing: Negative priming and related phenomena. In D. L. Medin (Ed.), The psychology of learning and motivation: Advances in research and theory (Vol. 38, pp. 1-44). San Diego, CA: Academic Press.

Neill, W. T., Valdes, L. A., \& Terry, K. M. (1995). Selective attention and the inhibitory control of cognition. In F. N. Dempster \& C. J. Brainerd (Eds.), Interference and inhibition in cognition (pp. 207261). San Diego, CA: Academic Press. https://doi.org/10.1016/ B978-012208930-5/50008-8

Tanaka, Y., \& Shimojo, S. (1996). Location vs. feature: Reaction time reveals dissociation between two visual functions. Vision Research, $36,2125-2140$.

Tanaka, Y., \& Shimojo, S. (2000). Repetition priming reveals sustained facilitation and transient inhibition in reaction time. Journal of Experimental Psychology: Human Perception and Performance, 26, 1421-1435. https://doi.org/10.1037/0096-1523.26.4.1421

Tipper, S. P., Brehaut, J. C., \& Driver, J. (1990). Selection of moving and static objects for the control of spatially directed action. Journal of Experimental Psychology: Human Perception and Performance, 16, 492-504. https://doi.org/10.1037/0096-1523.16.3.492

Vaughan, J. (1984). Saccades directed at previously attended locations in space. In A. J. Gale \& C. W. Johnson (Eds.), Theoretical and applied aspects of eye movement research (pp. 143-150). Amsterdam, The Netherlands: Elsevier.

Yashar, A., \& Lamy, D. (2010). Intertrial repetition affects perception: The role of focused attention. Journal of Vision, 10(14), 3:1-8. https://doi.org/10.1167/10.14.3 